\title{
LQG CONTROL WITH MISSING OBSERVATION AND CONTROL PACKETS
}

\author{
Bruno Sinopoli, Luca Schenato, Massimo Franceschetti, \\ Kameshwar Poolla, Shankar Sastry
}

\author{
Department of Electrical Engineering and Computer \\ Sciences \\ University of California at Berkeley \\ \{sinopoli,lusche,massimof,poolla,sastry\}@eecs.berkeley.edu
}

\begin{abstract}
The paper considers the Linear Quadratic Gaussian (LQG) optimal control problem in the discrete time setting and when data loss may occur between the sensors and the estimation-control unit and between the latter and the actuation points. For protocols where packets are acknowledged at the receiver (e.g. TCP type protocols), the separation principle holds. Moreover, the optimal LQG control is a linear function of the state. Finally, building upon our previous results on estimation with unreliable communication, the paper shows the existence of critical arrival probabilities below which the optimal controller fails to stabilize the system. This is done by providing analytic upper and and lower bounds on the cost functional, and stochastically characterizing their convergence properties in the infinite horizon. More interestingly, it turns out that when there is no feedback on whether a control packet has been delivered or not(e.g. UDP type protocols), the LQG optimal controller is in general nonlinear. A special case when the optimal controller is indeed linear is shown.
\end{abstract}

Keywords: LQG control, optimal control, linear control systems, stochastic control, distributed control

\section{INTRODUCTION}

Today, an increasingly growing number of applications demand remote control of plants over unreliable networks. These include wireless sensor networks used for estimation and control of dynamical systems (Sinopoli et al., 2003). In these systems issues of communication delay, data loss, and time synchronization between components play a key role. Communication and control become tightly coupled such that the two issues cannot be addressed independently.

Consider, for example, the problem of navigating a fleet of vehicles based on the estimate from a

\footnotetext{
1 Partially supported by the Defense Advanced Research Projects Agency under Grant F33615-01-C-1895
}

sensor web of its current position and velocity. The measurements underlying this estimate and control packets sent to the car from a supervisory controller can be lost or delayed due to the unreliability of the wireless links. What is the amount of data loss that the control loop can tolerate to reliably perform the navigation task? Can communication protocols be designed to satisfy this constraint? The goal of this paper is to examine some control-theoretic implications of using unreliable networks for control. These require a generalization of classical control techniques that explicitly take into account the stochastic nature of the communication channel.

Communication channels typically use one of two kinds of protocols: Transmission Control (TCP) or User Datagram (UDP). In the first case there is 




Fig. 1. Overview of the system. The study the statistical convergence of the expected state covariance of the discrete time LQG is performed, where both the observation and the control signal, travelling over an unreliable communication channel, can be lost at each time step with probability $1-\lambda$ and $1-\nu$ respectively.

acknowledgement of received packets, while in the second case no-feedback is provided on the communication link. This paper studies the effect of data losses due to the unreliability of the network links in these two cases. It generalizes the Linear Quadratic Gaussian (LQG) optimal control problem - modeling the arrival of an observation as a random process whose parameters are related to the characteristics of the communication channel. Accordingly, two independent Bernoulli processes are considered, of parameters $\bar{\gamma}$ and $\bar{\nu}$, that govern packet loss between the sensors and the estimation-control unit, and between the latter and the actuation points, see Figure 1.

It turns out that in the TCP case the classic separation principle holds and the optimal controller is a linear function of the state. However, in the UDP case, a counter-example shows that the optimal controller is in general non-linear. It is also shown that in the special case when the state is fully observable and the observation noise is zero the optimal controller is indeed linear. A similar, but slightly less general special case was previously analyzed by (Imer et al., 2004), considering not only the observation noise but also the process noise to be zero and the input coefficient matrix to be invertible.

A final set of results that are provided are on convergence in the infinite horizon. In this case, previous results on estimation with missing observation packets in (Sinopoli et al., 2004) are extended to the control case, showing the existence of critical values for the parameters of the Bernoulli arrival processes, below which a transition to instability occurs and the optimal controller fails to stabilize the system in both the TCP and the UDP settings. In other words, in order to have stability, the packet loss rate must be below a given threshold that depends on the dynamics of the system.

Finally, we want to mention some related work. Study of stability of dynamical systems where components are connected asynchronously via communication channels has received considerable attention in the past few years and our contribution can be put in the context of the previous literature.

In (Gupta et al., 2004), the authors proposed to place an estimator, i.e. a Kalman filter, at the sensor side of the link without assuming any statistical model for the data loss process. Other work includes Nilsson (Nilsson, 1998) that presents the LQG optimal regulator with bounded delays between sensors and controller, and between the controller and the actuator. In this work bounds for the critical probability values are not provided. Additionally, there is no analytic solution for the optimal controller. The case where dropped measurements are replaced by zeros is considered by Hadijcostis and Touri (Hadjicostis and Touri, 2002), in the scalar case. Other approaches include using the last received sample for control, or designing a dropout compensator (Ling and Lemmon, 2003b; Ling and Lemmon, 2003a), which combines in a single process estimation and control. This paper considers the alternative approach where the external compensator feeding the controller is the optimal time varying Kalman gain. Moreover, the proposed solution is analyzed in state space domain rather than in frequency domain as it was presented in (Ling and Lemmon, 2003a), and this paper considers the more general Multiple Input Multiple Output (MIMO) case.

The work of (Imer et al., 2004) is the closest to the present paper, which in addition considers the more general case when the matrix $C$ is not the identity and there is noise in the observation and in the process.

The paper is organized as follows. Section 2 will provide a mathematical formulation for the problem. Section 3 provides some preliminary results. Section 4 illustrates the TCP case, while the UDP case is studied in section 5. Finally conclusions and directions for future work are presented in

\section{PROBLEM FORMULATION}

Consider the following linear stochastic system with intermittent observations:

$$
\begin{aligned}
x_{k+1} & =A x_{k}+\nu_{k} B u_{k}+w_{k} \\
y_{k} & =C x_{k}+v_{k},
\end{aligned}
$$

where $\left(x_{0}, w_{k}, v_{k}\right)$ are Gaussian, uncorrelated, white, with mean $\left(\bar{x}_{0}, 0,0\right)$ and covariance $\left(P_{0}, Q, R_{k}\right)$ respectively, $R_{k}=\gamma_{k} R+\left(1-\gamma_{k}\right) \sigma^{2} I$, and $\left(\gamma_{k}, \nu_{k}\right)$ 
are i.i.d. Bernoulli random variable with $P\left(\gamma_{k}=\right.$ $1)=\bar{\gamma}$ and $P\left(\nu_{k}=1\right)=\bar{\nu}$. Let us define the following information sets:

$$
\mathcal{I}_{k}= \begin{cases}\mathcal{F}_{k} \triangleq\left\{\mathbf{y}^{k}, \boldsymbol{\gamma}^{k}, \nu^{k-1}\right\}, & \text { TCP comm. protocol } \\ \mathcal{G}_{k} \triangleq\left\{\mathbf{y}^{k}, \boldsymbol{\gamma}^{k}\right\}, & \text { UDP comm. protocol }\end{cases}
$$

where $\mathbf{y}^{k}=\left(y_{k}, y_{k-1}, \ldots, y_{1}\right), \gamma^{k}=\left(\gamma_{k}, \gamma_{k-1}, \ldots, \gamma_{1}\right)$, and $\boldsymbol{\nu}^{k}=\left(\nu_{k}, \nu_{k-1}, \ldots, \nu_{1}\right)$.

Consider also the following cost function:

$$
\begin{aligned}
& J_{N}\left(\mathbf{u}^{N-1}\right)= \\
& =\mathbb{E}\left[x_{N}^{\prime} W_{N} x_{N}+\sum_{k=0}^{N-1}\left(x_{k}^{\prime} W_{k} x_{k}+\nu_{k} u_{k}^{\prime} U_{k} u_{k}\right) \mid \mathcal{I}_{N}\right] .
\end{aligned}
$$

Note that we are weighting the input only if it is successfully received at the plant. In fact, if it is not received, the plant applies zero input and therefore there is no energy expenditure.

We now look for a control input sequence $\mathbf{u}^{* N-1}$ as a function of the admissible information set $\mathcal{I}_{k}$, i.e.

$$
u_{k}=g_{k}\left(I_{k}\right)
$$

that minimizes the functional defined in Equation (4), i.e.

$$
J_{N}^{*} \triangleq \min _{\mathbf{u}^{N-1}} J_{N}\left(\mathbf{u}^{N-1}\right)=J_{N}\left(\mathbf{u}^{* N-1}\right),
$$

where $\mathcal{I}_{k}=\left\{\mathcal{F}_{k}, \mathcal{G}_{k}\right\}$ is one of the sets defined in Equation 3. The set $\mathcal{F}$ corresponds to the information provided under TCP communication protocol in which successful or unsuccessful packet delivery at the receiver is acknowledged to the sender. The set $\mathcal{G}$ corresponds to the information provided under UDP communication protocol in which the sender receives no information about the delivery of the transmitted packet to the receiver. This protocol scheme is simpler to implement than TCP from a communication standpoint, however the price to pay is a less rich set of information. The goal of this paper is to design optimal LQG controllers for each of these protocols for a general discrete-time linear stochastic system.

\section{MATHEMATICAL BACKGROUND}

Before proceeding, let us define the following variables:

$$
\begin{aligned}
& \hat{x}_{k \mid k} \triangleq \mathbb{E}\left[x_{k} \mid \mathcal{I}_{k}\right], \\
& e_{k \mid k} \triangleq x_{k}-\hat{x}_{k \mid k}, \\
& P_{k \mid k} \triangleq \mathbb{E}\left[e_{k \mid k} e_{k \mid k}^{\prime} \mid \mathcal{I}_{k}\right] .
\end{aligned}
$$

Derivations below will make use of the following facts:

Lemma 1. The following facts are true:

(a) $\mathbb{E}\left[\left(x_{k}-\hat{x}_{k}\right) \hat{x}_{k}^{\prime} \mid \mathcal{I}_{k}\right]=\mathbb{E}\left[e_{k \mid k} \hat{x}_{k}^{\prime} \mid \mathcal{I}_{k}\right]=0$

(b) $\mathbb{E}\left[x_{k}^{\prime} S x_{k} \mid \mathcal{I}_{k}\right]=\hat{x}_{k}^{\prime} S \hat{x}_{k}+\operatorname{trace}\left(S P_{k \mid k}\right)=$ $\hat{x}_{k}^{\prime} S \hat{x}_{k}+\mathbb{E}\left[e_{k}^{\prime} S e_{k} \mid \mathcal{I}_{k}\right], \quad \forall S$ (c) $\mathbb{E}\left[\mathbb{E}\left[g\left(x_{k+1}\right) \mid \mathcal{I}_{k+1}\right] \mid \mathcal{I}_{k}\right]=\mathbb{E}\left[g\left(x_{k+1}\right) \mid \mathcal{I}_{k}\right], \forall g(\cdot)$.

Use of the following properties will prove to be useful when deriving the equation for the optimal LQG controller. Let us compute the following expectation:

$$
\begin{aligned}
\mathbb{E} & {\left[x_{k+1}^{\prime} S x_{k+1} \mid \mathcal{I}_{k}\right]=} \\
= & \mathbb{E}\left[\left(A x_{k}+\nu_{k} B u_{k}+w_{k}\right)^{\prime} S\left(A x_{k}+\nu_{k} B u_{k}+w_{k}\right) \mid \mathcal{I}_{k}\right] \\
= & \mathbb{E}\left[x_{k}^{\prime} A^{\prime} S A x_{k} \mid \mathcal{I}_{k}\right]+\bar{\nu} u_{k}^{\prime} B^{\prime} S B u_{k}+ \\
& +2 \bar{\nu} u_{k}^{\prime} B^{\prime} S A \hat{x}_{k \mid k}+\operatorname{trace}(S Q)
\end{aligned}
$$

where both the independence of $\nu_{k}, w_{k}, x_{k}$, and the zero-mean property of $w_{k}$ are exploited. The previous expectation holds true for both the information sets $\mathcal{I}_{k}=\left\{\mathcal{F}_{k}, \mathcal{G}_{k}\right\}$. Also

$$
\begin{aligned}
\mathbb{E}\left[e_{k \mid k}^{\prime} T e_{k \mid k} \mid \mathcal{I}_{k}\right] & =\operatorname{trace}\left(T \mathbb{E}\left[e_{k \mid k} e_{k \mid k}^{\prime} \mid \mathcal{I}_{k}\right]\right)= \\
& =\operatorname{trace}\left(T P_{k \mid k}\right) .
\end{aligned}
$$

\section{TCP}

First, equations for the optimal estimator are derived. They will be needed to solve the LQG controller design problem, as it will be shown later.

\subsection{Estimator Design, $\sigma \rightarrow+\infty$}

Equations for optimal estimator are derived using similar arguments used for the standard Kalman filtering equations. The innovation step is given by:

$$
\begin{aligned}
\hat{x}_{k+1 \mid k} & \triangleq \mathbb{E}\left[x_{k+1} \mid \nu_{k}, \mathcal{F}_{k}\right]=A \hat{x}_{k \mid k}+\nu_{k} B u_{k} \\
e_{k+1 \mid k} & \triangleq x_{k+1}-\hat{x}_{k+1 \mid k}=A e_{k \mid k}+w_{k} \\
P_{k+1 \mid k} & \triangleq \mathbb{E}\left[e_{k+1 \mid k} e_{k+1 \mid k}^{\prime} \mid \nu_{k}, \mathcal{F}_{k}\right]=A P_{k \mid k} A^{\prime}+Q,(12)
\end{aligned}
$$

where the independence of $w_{k}$ and $\mathcal{F}_{k}$ is exploited. Since $y_{k+1}, \gamma_{k+1}, w_{k}$ and $\mathcal{F}_{k}$ are all independent of each other and following the same approach described in (Sinopoli et al., 2004), the correction step is given by:

$$
\begin{aligned}
\hat{x}_{k+1 \mid k+1} & =\hat{x}_{k+1 \mid k}+\gamma_{k+1} K_{k+1}\left(y_{k+1}-C \hat{x}_{k+1 \mid k}\right) \\
e_{k+1 \mid k+1} & \triangleq x_{k+1}-\hat{x}_{k+1 \mid k+1} \\
& =\left(I-\gamma_{k+1} K_{k+1} C\right) e_{k+1 \mid k}-\gamma_{k+1} K_{k+1} v_{k+1} \\
P_{k+1 \mid k+1} & =P_{k+1 \mid k}-\gamma_{k+1} K_{k+1} C P_{k+1 \mid k} \\
K_{k+1} & \triangleq P_{k+1 \mid k} C^{\prime}\left(C P_{k+1 \mid k} C^{\prime}+R\right)^{-1}
\end{aligned}
$$

after taking the limit $\sigma \rightarrow+\infty$. The initial conditions for the estimator iterative equations are $\hat{x}_{0 \mid-1}=0$ and $P_{0 \mid-1}=P_{0}$. 


\subsection{Controller design}

Derivation of the optimal feedback control law and the corresponding value for the objective function will follow the dynamic programming approach based on the cost-to-go iterative procedure.

Define the optimal value function $V_{k}\left(x_{k}\right)$ as follows:

$$
\begin{gathered}
V_{N}\left(x_{N}\right) \triangleq \mathbb{E}\left[x_{N}^{\prime} W_{N} x_{N} \mid \mathcal{F}_{N}\right] \\
V_{k}\left(x_{k}\right) \triangleq \min _{u_{k}} \mathbb{E}\left[x_{k}^{\prime} W_{k} x_{k}+\nu_{k} u_{k}^{\prime} U_{k} u_{k}+V_{k+1}\left(x_{k+1}\right) \mid \mathcal{F}_{k}\right]
\end{gathered}
$$

Using dynamic programming theory (Kumar and Varaiya, 1986) (Bertsekas and Tsitsiklis, 1996), one can show that $J_{N}^{*}=V_{0}\left(x_{0}\right)$. We claim that the value function $V_{k}\left(x_{k}\right)$ can be written as:

$$
V_{k}\left(x_{k}\right)=\mathbb{E}\left[x_{k}^{\prime} S_{k} x_{k} \mid \mathcal{F}_{k}\right]+c_{k}, \quad k=0, \ldots, N
$$

where the matrix $S_{k}$ and the scalar $c_{k}$ are to be determined and are independent of the information set $\mathcal{F}$. The proof follows an induction argument. The claim is certainly true for $k=N$ with the choice of parameters $S_{N}=W_{N}$ and $c_{N}=$ 0 . Suppose now that the claim is true for $k+1$, i.e. $V_{k+1}\left(x_{k+1}\right)=\mathbb{E}\left[x_{k+1}^{\prime} S_{k+1} x_{k+1} \mid \mathcal{F}_{k+1}\right]+c_{k+1}$. The value function at time step $k$ is the following:

$$
\begin{aligned}
& V_{k}\left(x_{k}\right)= \\
& =\min _{u_{k}} \mathbb{E}\left[x_{k}^{\prime} W_{k} x_{k}+\nu_{k} u_{k}^{\prime} U_{k} u_{k}+V_{k+1}\left(x_{k+1}\right) \mid \mathcal{I}_{k}\right] \\
& =\min _{u_{k}} \mathbb{E}\left[x_{k}^{\prime} W_{k} x_{k}+\nu_{k} u_{k}^{\prime} U_{k} u_{k}+\right. \\
& \left.+\mathbb{E}\left[x_{k+1}^{\prime} S_{k+1} x_{k+1}+c_{k+1} \mid \mathcal{F}_{k+1}\right] \mid \mathcal{I}_{k}\right] \\
& =\min _{u_{k}} \mathbb{E}\left[x_{k}^{\prime} W_{k} x_{k}+\nu_{k} u_{k}^{\prime} U_{k} u_{k}+x_{k+1}^{\prime} S_{k+1} x_{k+1}+\right. \\
& \left.+c_{k+1} \mid \mathcal{I}_{k}\right] \\
& =\mathbb{E}\left[x_{k}^{\prime} W_{k} x_{k}+x_{k}^{\prime} A^{\prime} S_{k+1} A x_{k} \mid \mathcal{I}_{k}\right]+ \\
& +\operatorname{trace}\left(S_{k+1} Q\right)+\mathbb{E}\left[c_{k+1} \mid \mathcal{I}_{k}\right]+ \\
& +\bar{\nu} \min _{u_{k}}\left(u_{k}^{\prime}\left(U_{k}+B^{\prime} S_{k+1} B\right) u_{k}+2 u_{k}^{\prime} B^{\prime} S_{k+1} A \hat{x}_{k \mid k}\right)
\end{aligned}
$$

where we used Lemma 1(c) in the third line, and Equation (8) in the last two lines. The value function is a quadratic function of the input, therefore the minimizer can be simply obtained by solving $\frac{\partial V_{k}}{\partial u_{k}}=0$, which gives:

$u_{k}=-\left(B^{\prime} S_{k+1} B+U_{k}\right)^{-1} B^{\prime} S_{k+1} A \hat{x}_{k \mid k}=L_{k} \hat{x}_{k \mid k}$.

The optimal feedback is thus a simple linear feedback of the estimated state. If we substitute the minimizer back into Equation (18), and we use the Equation (17) we get:

$$
\begin{array}{lr}
V_{k}\left(x_{k}\right)= & \text { it } \\
=\mathbb{E}\left[x_{k}^{\prime} W_{k} x_{k}+x_{k}^{\prime} A^{\prime} S_{k+1} A x_{k} \mid \mathcal{I}_{k}\right]+\operatorname{trace}\left(S_{k+1} Q\right)+ & \text { ar } \\
+\mathbb{E}\left[c_{k+1} \mid \mathcal{I}_{k}\right]-\bar{\nu} \hat{x}_{k \mid k}^{\prime} A^{\prime} S_{k+1} B\left(U_{k}+B^{\prime} S_{k+1} B\right)^{-1} B^{\prime} S_{k+1} A \hat{x}_{k \mid k}
\end{array}
$$

and we have:

$$
\begin{aligned}
& \mathbb{E}\left[x_{k}^{\prime} S_{k} x_{k} \mid \mathcal{I}_{k}\right]+c_{k}= \\
& =\mathbb{E}\left[x_{k}^{\prime} W_{k} x_{k}+x_{k}^{\prime} A^{\prime} S_{k+1} A x_{k}-\right. \\
& \left.+\bar{\nu} x_{k}^{\prime} A^{\prime} S_{k+1} B\left(U_{k}+B^{\prime} S_{k+1} B\right)^{-1} B^{\prime} S_{k+1} A x_{k} \mid \mathcal{I}_{k}\right]+ \\
& +\operatorname{trace}\left(S_{k+1} Q\right)+\mathbb{E}\left[c_{k+1} \mid \mathcal{I}_{k}\right]+ \\
& +\bar{\nu} \operatorname{trace}\left(A^{\prime} S_{k+1} B\left(U_{k}+B^{\prime} S_{k+1} B\right)^{-1} B^{\prime} S_{k+1} P_{k \mid k}\right)(21)
\end{aligned}
$$

where we used Lemma 1(b). For the previous equation to hold for all $x_{k}$, we need to have:

$$
\begin{aligned}
S_{k} & =A^{\prime} S_{k+1} A+W_{k}- \\
& +\bar{\nu} A^{\prime} S_{k+1} B\left(B^{\prime} S_{k+1} B+U_{k}\right)^{-1} B^{\prime} S_{k+1} A \\
c_{k} & =\bar{\nu} \operatorname{trace}\left(A^{\prime} S_{k+1} B\left(U_{k}+B^{\prime} S_{k+1} B\right)^{-1} B^{\prime} S_{k+1} P_{k \mid k}\right)+ \\
& +\operatorname{trace}\left(S_{k+1} Q\right)+\mathbb{E}\left[c_{k+1} \mid \mathcal{I}_{k}\right] \\
& =\operatorname{trace}\left(\left(A^{\prime} S_{k+1} A+W_{k}-S_{k}\right) P_{k \mid k}\right)+ \\
& +\operatorname{trace}\left(S_{k+1} Q\right)+\mathbb{E}\left[c_{k+1} \mid \mathcal{I}_{k}\right]
\end{aligned}
$$

Therefore, the cost function for the optimal LQG using TCP is given by:

$$
\begin{aligned}
J_{N}^{*} & =V_{0}\left(x_{0}\right)=\mathbb{E}\left[x_{0}^{\prime} S_{0} x_{0}\right]+ \\
& +\sum_{k=0}^{N-1}\left(\operatorname{trace}\left(\left(A^{\prime} S_{k+1} A+W_{k}-S_{k}\right) \mathbb{E}_{\gamma}\left[P_{k \mid k}\right]\right)+\right. \\
& \left.+\operatorname{trace}\left(S_{k+1} Q\right)\right) \\
& =\bar{x}_{0}^{\prime} S_{0} \bar{x}_{0}+\operatorname{trace}\left(S_{0} P_{0}\right)+ \\
& +\sum_{k=0}^{N-1}\left(\operatorname{trace}\left(\left(A^{\prime} S_{k+1} A+W_{k}-S_{k}\right) \mathbb{E}_{\gamma}\left[P_{k \mid k}\right]\right)+\right. \\
& \left.+\operatorname{trace}\left(S_{k+1} Q\right)\right) .
\end{aligned}
$$

The matrices $\left\{P_{k \mid k}\right\}_{k=0}^{N}$ are stochastic since they are function of the sequence $\left\{\gamma_{k}\right\}$. The exact expected value of these matrices cannot be computed analytically, since they are nonlinear function of the arrival sequence $\gamma_{k}$, as shown in (Sinopoli et al., 2004). However, they can bounded by computable deterministic quantities. In fact let us consider the following equations:

$$
\begin{aligned}
\widehat{P}_{k+1 \mid k} & =A \widehat{P}_{k \mid k-1} A^{\prime}+Q- \\
& +\bar{\gamma} A \widehat{P}_{k \mid k-1} C^{\prime}\left(C \widehat{P}_{k \mid k-1} C^{\prime}+R\right)^{-1} C \widehat{P}_{k \mid k-1} A^{\prime} \\
\widehat{P}_{k \mid k} & =\widehat{P}_{k \mid k-1}-\bar{\gamma} \widehat{P}_{k \mid k-1} C^{\prime}\left(C \widehat{P}_{k \mid k-1} C^{\prime}+R\right)^{-1} C \widehat{P}_{k \mid k}(26) \\
\widetilde{P}_{k+1 \mid k} & =(1-\bar{\gamma}) A \widetilde{P}_{k \mid k-1} A^{\prime}+Q \\
\widetilde{P}_{k \mid k} & =(1-\bar{\gamma}) \widetilde{P}_{k \mid k-1}
\end{aligned}
$$

initialized to $\widehat{P}_{0 \mid-1}=\widetilde{P}_{0 \mid-1}=P_{0}$. Using similar arguments as those in (Sinopoli et al., 2004), it is possible to show that the matrices $P_{k \mid k}$ 's are concave and monotonic functions of $P_{k \mid k-1}$. Therefore, the following bounds are true:

$$
\widetilde{P}_{k \mid k} \leq \mathbb{E}_{\gamma}\left[P_{k \mid k}\right] \leq \widehat{P}_{k \mid k},
$$




$$
\begin{aligned}
J_{N}^{\min } & \leq J_{N}^{*} \leq J_{N}^{\max } \\
J_{N}^{\max } & =\bar{x}_{0}^{\prime} S_{0} \bar{x}_{0}+\operatorname{trace}\left(S_{0} P_{0}\right)+ \\
& +\sum_{k=0}^{N-1}\left(\operatorname{trace}\left(\left(A^{\prime} S_{k+1} A+W_{k}-S_{k}\right) \widehat{P}_{k \mid k}\right)+\right. \\
& \left.+\operatorname{trace}\left(S_{k+1} Q\right)\right) \\
J_{N}^{\min } & =\bar{x}_{0}^{\prime} S_{0} \bar{x}_{0}+\operatorname{trace}\left(S_{0} P_{0}\right)+ \\
& +\sum_{k=0}^{N-1}\left(\operatorname{trace}\left(\left(A^{\prime} S_{k+1} A+W_{k}-S_{k}\right) \widetilde{P}_{k \mid k}\right)\right. \\
& \left.+\operatorname{trace}\left(S_{k+1} Q\right)\right)
\end{aligned}
$$

\subsection{Finite and Infinite Horizon LQG control}

The previous equations were derived for the finite horizon LQG. The infinite horizon LQG can be obtained by taking the limit for $N \rightarrow+\infty$ of the previous equations. However, the matrices $\left\{P_{k \mid k}\right\}$ depend on the specific realization of the observation sequence $\left\{\gamma_{k}\right\}$, therefore the minimal cost $J_{N}$ is a stochastic function and does not have a limit. Differently from standard LQG controller design where the controller always stabilizes the original system, in the case of control packet loss, the stability can be lost if the arrival probability $\bar{\nu}, \bar{\gamma}$ is below a certain threshold. In particular the equation for the cost matrix $S_{k}$ is the solution of a Modified Riccati Algebraic Equation (MARE) which was already introduced and studied in (Sinopoli et al., 2004). In particular, Equation (22) is the dual of the estimator equation presented in (Sinopoli et al., 2004). Therefore, the same conclusions can be drawn and the previous result can be summarized in the following theorem:

Theorem 2. (Finite Horizon LQG under TCP). Consider the system (1)-(2) and consider the problem of minimizing the cost function (4) with policy $u_{k}=f\left(\mathcal{F}_{k}\right)$, where $\mathcal{F}_{k}$ is the information available under TCP communication, given in Equation (3). Then, the optimal control is a linear function of the estimated system state given by Equation (19), where the matrix $S_{k}$ can be computed iteratively using Equation (22). The separation principle still holds under TCP communication, since the optimal estimator is independent of the control input $u_{k}$. The optimal state estimator is given by Equations (10)-(13) and (12)-(16), and the minimal achievable cost is given by Equation (24).

Theorem 3. (Infinite Horizon LQG under TCP). Consider the same systems as defined in the previous theorem with the following additional hypothesis: $W_{N}=W_{k}=W$ and $U_{k}=U$. Moreover, let $(A, B)$ and $\left(A, Q^{\frac{1}{2}}\right)$ be stabilizable, and let $(A, C)$ and $\left(A, W^{\frac{1}{2}}\right)$ be detectable. Let us consider the limiting case $N \rightarrow+\infty$. There exist critical arrival probabilities $\nu_{\min }$ and $\gamma_{\min }$ which satisfy the following property:

$$
\begin{aligned}
& \min \left(1,1-\frac{1}{\left|\lambda_{\max }(A)\right|^{2}}\right) \leq \nu_{\min } \leq 1, \\
& \min \left(1,1-\frac{1}{\left|\lambda_{\max }(A)\right|^{2}}\right) \leq \gamma_{\min } \leq 1,
\end{aligned}
$$

where $\left|\lambda_{\max }(A)\right|$ is the eigenvalue of matrix $A$ with largest absolute value, such that for all $\bar{\nu}>$ $\nu_{\min }$ and $\bar{\gamma}>\gamma_{\min }$ we have:

$$
\begin{aligned}
& L_{k}=L_{\infty}=-\left(B^{\prime} S_{\infty} B+U\right)^{-1} B^{\prime} S_{\infty} A \\
& \frac{1}{N} J_{N}^{m i n} \leq \frac{1}{N} J_{N}^{*} \leq \frac{1}{N} J_{N}^{\max }
\end{aligned}
$$

where the mean cost bounds $J_{N}^{\min }, J_{N}^{\max }$ are given by:

$$
\begin{aligned}
J_{\infty}^{\max } & =\lim _{N \rightarrow+\infty} \frac{1}{N} J_{N}^{\max } \\
& =\operatorname{trace}\left(( A ^ { \prime } S _ { \infty } A + W _ { k } - S _ { \infty } ) \left(\widehat{P}_{\infty}-\right.\right. \\
& \left.\left.+\bar{\gamma} \widehat{P}_{\infty} C^{\prime}\left(C \widehat{P}_{\infty} C^{\prime}+R\right)^{-1} C \widehat{P}_{\infty}\right)\right)+\operatorname{trace}\left(S_{\infty} Q\right) \\
J_{\infty}^{\text {min }} & =\lim _{N \rightarrow+\infty} \frac{1}{N} J_{N}^{\text {min }} \\
& =(1-\bar{\gamma}) \operatorname{trace}\left(\left(A^{\prime} S_{\infty} A+W_{k}-S_{\infty}\right) \widetilde{P}_{\infty}\right)+ \\
& +\operatorname{trace}\left(S_{\infty} Q\right)
\end{aligned}
$$

and the matrices $S_{\infty}, \bar{P}_{\infty}, \underline{P}_{\infty}$ are:

$$
\begin{aligned}
S_{\infty} & =A^{\prime} S_{\infty} A+W-\bar{\nu} A^{\prime} S_{\infty} B\left(B^{\prime} S_{\infty} B+U\right)^{-1} B^{\prime} S_{\infty} A \\
\bar{P}_{\infty} & =A \bar{P} \bar{P}_{\infty} A^{\prime}+Q-\bar{\gamma} A \bar{P}{ }_{\infty} C^{\prime}\left(C \bar{P}_{\infty} C^{\prime}+R\right)^{-1} C \bar{P}_{\infty} A^{\prime} \\
\underline{P}_{\infty} & =(1-\bar{\gamma}) A \underline{P}_{\infty} A^{\prime}+Q
\end{aligned}
$$

Moreover, the assumptions above are necessary and sufficient conditions for boundedness of the cost function under LQG feedback. The critical probabilities $\nu_{\min }$ and $\gamma_{\min }$ can be computed via the solution of the following LMIs optimization problems:

$$
\gamma_{\text {min }}=\operatorname{argmin}_{\bar{\gamma}} \Psi_{\gamma}(Y, Z)>0, \quad 0 \leq Y \leq I .
$$

$$
\begin{aligned}
& \Psi_{\gamma}(Y, Z)= \\
& =\left[\begin{array}{ccc}
Y & \sqrt{\gamma}(Y A+Z C) & \sqrt{1-\gamma} Y A \\
\sqrt{\gamma}\left(A^{\prime} Y+C^{\prime} Z^{\prime}\right) & Y & 0 \\
\sqrt{1-\gamma} A^{\prime} Y & 0 & Y
\end{array}\right] \\
& \nu_{\text {min }}=\operatorname{argmin}_{\bar{\nu}} \Psi_{\nu}(Y, Z)>0, \quad 0 \leq Y \leq I . \\
& \Psi_{\nu}(Y, Z)= \\
& =\left[\begin{array}{ccc}
Y & \sqrt{\nu}\left(Y A^{\prime}+Z B^{\prime}\right) & \sqrt{1-\nu} Y A^{\prime} \\
\sqrt{\nu}\left(A Y+B Z^{\prime}\right) & Y & 0 \\
\sqrt{1-\nu} A Y & 0 & Y
\end{array}\right]
\end{aligned}
$$




\section{UDP}

In this section equations for the optimal estimator and controller design for case of UDP communication protocol are derived. The UDP case corresponds to the information set $G_{k}$, as defined in Equation (3). Some of the derivations are analogous to the previous section and are therefore skipped.

\subsection{Estimator Design, $\sigma \rightarrow+\infty$}

We derive the equations for optimal estimator using similar arguments to the standard Kalman filtering equations. The innovation step is given by:

$$
\begin{aligned}
\hat{x}_{k+1 \mid k} & \triangleq \mathbb{E}\left[x_{k+1} \mid \mathcal{G}_{k}\right]=\mathbb{E}\left[A x_{k}+\nu_{k} B u_{k}+w_{k} \mid \mathcal{G}_{k}\right] \\
& =A \mathbb{E}\left[x_{k} \mid \mathcal{G}_{k}\right]+\mathbb{E}\left[\nu_{k}\right] B u_{k} \\
& =A \hat{x}_{k \mid k}+\bar{\nu} B u_{k} \\
e_{k+1 \mid k} & \triangleq x_{k+1}-\hat{x}_{k+1 \mid k} \\
P_{k+1 \mid k} & \triangleq \mathbb{E}\left[e_{k+1 \mid k} e_{k+1 \mid k}^{\prime} \mid \mathcal{G}_{k}\right] \\
& =A P_{k \mid k} A^{\prime}+Q+\bar{\nu}(1-\bar{\nu}) B u_{k} u_{k}^{\prime} B^{\prime}
\end{aligned}
$$

where we used the independence and zero-mean of $w_{k},\left(\nu_{k}-\bar{\nu}\right)$, and $\mathcal{G}_{k}$. Note how under UDP communication protocol, differently from TCP communication, the error covariance $P_{k+1 \mid k}$ depends explicitly on the control input $u_{k}$. This is the main difference with TCP.

The correction step is the same as for the TCP case, given by:

$$
\begin{aligned}
\hat{x}_{k+1 \mid k+1} & =\hat{x}_{k+1 \mid k}+\gamma_{k+1} K_{k+1}\left(y_{k+1}-C \hat{x}_{k+1 \mid k}\right) \\
P_{k+1 \mid k+1} & =P_{k+1 \mid k}-\gamma_{k+1} K_{k+1} C P_{k+1 \mid k}, \\
K_{k+1} & \triangleq P_{k+1 \mid k} C^{\prime}\left(C P_{k+1 \mid k} C^{\prime}+R\right)^{-1},
\end{aligned}
$$

where again we took the limit $\sigma \rightarrow+\infty$.

The initial conditions for the estimator iterative equations are:

$$
\begin{aligned}
& \hat{x}_{0 \mid-1}=0 \\
& P_{0 \mid-1}=P_{0}
\end{aligned}
$$

\subsection{Controller design: General case}

In this section, we show that the optimal LQG controller is, in general, not a linear function of the state estimate, since estimator and controller design cannot be separated anymore. To show this, we consider a simple scalar system and we proceed using the dynamic programming approach. Let us consider an unstable scalar system where $A=1, B=1, C=1, W_{N}=W_{k}=1, U_{k}=$ $0, R=1, Q=0$. Similarly to the TCP case, the value function, $V_{k}\left(x_{k}\right)$ for $k=N$ is given by $V_{N}\left(x_{N}\right)=\mathbb{E}\left[x_{N}^{\prime} W_{N} x_{N} \mid \mathcal{G}_{N}\right]=\mathbb{E}\left[x_{N}^{2} \mid \mathcal{G}_{N}\right]$. Let us consider the value function for $k=N-1$ :

$$
\begin{aligned}
& V_{N-1}\left(x_{N-1}\right)= \\
& =\min _{u_{N-1}} \mathbb{E}\left[x_{N-1}^{2}+V_{N}\left(x_{N}\right) \mid \mathcal{G}_{N-1}\right] \\
& =\min _{u_{N-1}} \mathbb{E}\left[x_{N-1}^{2}+x_{N}^{2} \mid \mathcal{G}_{N-1}\right] \\
& =\min _{u_{N-1}}\left(\mathbb{E}\left[x_{N-1}^{2}+x_{N-1}^{2} \mid \mathcal{G}_{N-1}\right]+\right. \\
& \left.+\bar{\nu} u_{N-1}^{2}+2 \bar{\nu} u_{N-1} \hat{x}_{N-1 \mid N-1}\right) \\
& =2 \mathbb{E}\left[x_{N-1}^{2} \mid \mathcal{G}_{N-1}\right]-\bar{\nu} \hat{x}_{N-1 \mid N-1}^{2} \\
& =(2-\bar{\nu}) \mathbb{E}\left[x_{N-1}^{2} \mid \mathcal{G}_{N-1}\right]+\bar{\nu} P_{N-1 \mid N-1}
\end{aligned}
$$

where we used Equation (8) in line (44), we substituted the minimizer $u_{N-1}^{*}$, given by:

$$
u_{N-1}^{*}=-\hat{x}_{N-1 \mid N-1}
$$

, in line (45), and we used Lemma 1(b) in line (46). Let us continue considering the value function for $k=N-2$ :

$$
\begin{aligned}
& V_{N-2}\left(x_{N-2}\right)= \\
& =\min _{u_{N-2}} \mathbb{E}\left[x_{N-2}^{2}+V_{N-1}\left(x_{N-1}\right) \mid \mathcal{G}_{N-2}\right] \\
& =\mathbb{E}\left[(3-\bar{\nu}) x_{N-2}^{2} \mid \mathcal{G}_{N-2}\right]+\bar{\gamma}+\bar{\nu} P_{N-2 \mid N-2}+ \\
& +\bar{\nu}(1-\bar{\gamma}) P_{N-2 \mid N-2}+ \\
& +\min _{u_{N-2}}\left(\bar{\nu}(2-\bar{\nu}) u_{N-2}^{2}+2 \bar{\nu}(2-\bar{\nu}) u_{N-2} \hat{x}_{N-2 \mid N-2}\right. \\
& +\bar{\nu}^{2}(1-\bar{\nu})(1-\bar{\gamma}) u_{N-2}^{2}+ \\
& \left.+\bar{\nu} \bar{\gamma} \frac{1}{P_{N-2 \mid N-2}+\bar{\nu}(1-\bar{\nu}) u_{N-2}^{2}+1}\right)
\end{aligned}
$$

The first three terms inside the round parenthesis are convex quadratic functions of the control input $u_{N-2}$, however the last term is not. Therefore, the minimizer $u_{N-2}^{*}$ is, in general, a non-linear function of the information set $\mathcal{G}_{k}$. We can summarize this result in the following theorem:

Theorem 4. Let us consider the stochastic system defined in Equations (1) with horizon $N \geq 2$. Then, the optimal control feedback $u_{k}=g_{k}^{*}\left(\mathcal{G}_{k}\right)$ that minimizes the functional (4) under UDP is, in general, a nonlinear function of information set $\mathcal{G}_{k}$.

PROOF. The proof can be given by showing the previous counter-example, where even a simple scalar system does not have a linear solution.

The nonlinearity of the input feedback arises from the fact that the correction error covariance matrix $P_{k+1 \mid k+1}$ is a non-linear function of the innovation error covariance $P_{k+1 \mid k}$. The only case when $P_{k+1 \mid k+1}$ is linear occurs when $R=0$ and $C$ is invertible, i.e. when it is possible to measure the state $x_{k}$ without noise. This special case is considered in the next section. 


\subsection{Controller design: $R=0$ and $C$ invertible}

Without loss of generality we can assume $C=I$, otherwise the linear transformation $y=C x$ would give an equivalent state space representation of the system for which $C=I$. Let us now consider the case when there is no measurement noise and we can measure the state $x_{k}$ when an observation packet is delivered.

In this case the estimator equations simplify as follows:

$$
\begin{aligned}
& K_{k+1}=I \\
& P_{k+1 \mid k+1}=\left(1-\gamma_{k+1}\right) P_{k+1 \mid k}= \\
& =\left(1-\gamma_{k+1}\right)\left(A^{\prime} P_{k \mid k} A+Q+\bar{\nu}(1-\bar{\nu}) B u_{k} u_{k}^{\prime} B^{\prime}\right) \\
& \mathbb{E}\left[P_{k+1 \mid k+1} \mid \mathcal{G}_{k}\right]= \\
& =(1-\bar{\gamma})\left(A^{\prime} P_{k \mid k} A+Q+\bar{\nu}(1-\bar{\nu}) B u_{k} u_{k}^{\prime} B^{\prime}\right)
\end{aligned}
$$

where we used independence of $\gamma_{k+1}$ and $P_{k \mid k}$ with respect to $\mathcal{G}_{k}$ in the last line. Similarly to the TCP case, we claim that the value function $V_{k}\left(x_{k}\right)$ can be written as follows:

$$
V_{k}\left(x_{k}\right)=\mathbb{E}\left[x_{k}^{\prime} S_{k} x_{k} \mid \mathcal{G}\right]+\operatorname{trace}\left(T_{k} P_{k \mid k}\right)+\operatorname{trace}\left(D_{k} Q\right)
$$

for $k=0, \ldots, N$. This is clearly true for $k=N$, since $V_{N}\left(x_{N}\right)=\mathbb{E}\left[x_{N}^{\prime} W_{N} x_{N} \mid \mathcal{G}_{N}\right]$. Therefore:

$$
S_{N}=W_{N}, \quad T_{N}=0, \quad D_{N}=0 .
$$

Let us suppose that the claim (48) is true for $k+1$ and let us show by induction it holds true for $k$.

$$
\begin{aligned}
& V_{k}\left(x_{k}\right)=\min _{u_{k}} \mathbb{E}\left[x_{k}^{\prime} W_{k} x_{k}+\nu_{k} u_{k}^{\prime} U_{k} u_{k}+V_{k+1}\left(x_{k+1}\right) \mid \mathcal{G}_{k}\right] \\
& =\min _{u_{k}}\left(\mathbb{E}\left[x_{k}^{\prime} W_{k} x_{k} \mid \mathcal{G}_{k}\right]+\bar{\nu} u_{k}^{\prime} U_{k} u_{k}+\mathbb{E}\left[x_{k+1}^{\prime} S_{k+1} x_{k+1}+\right.\right. \\
& \left.\left.+\operatorname{trace}\left(T_{k+1} P_{k+1 \mid k+1}\right)+\operatorname{trace}\left(D_{k+1} Q\right) \mid \mathcal{G}_{k}\right]\right) \\
& =\mathbb{E}\left[x_{k}^{\prime}\left(W_{k}+A^{\prime} S_{k+1} A\right) x_{k} \mid \mathcal{G}_{k}\right]+ \\
& +(1-\bar{\gamma}) \operatorname{trace}\left(T_{k+1}\left(A^{\prime} P_{k \mid k} A+Q\right)\right)+\operatorname{trace}\left(D_{k+1} Q\right) \\
& +\min _{u_{k}}\left(\bar{\nu} u_{k}^{\prime} U_{k} u_{k}+\bar{\nu} u_{k}^{\prime} B^{\prime} S_{k+1} B u_{k}+2 \bar{\nu} u_{k}^{\prime} B^{\prime} S_{k+1} A \hat{x}_{k \mid k}+\right. \\
& \left.+\bar{\nu}(1-\bar{\nu})(1-\bar{\gamma}) \operatorname{trace}\left(T_{k+1} B u_{k} u_{k}^{\prime} B^{\prime}\right)\right) \\
& =\mathbb{E}\left[x_{k}^{\prime}\left(W_{k}+A^{\prime} S_{k+1} A\right) x_{k} \mid \mathcal{G}_{k}\right]+ \\
& +(1-\bar{\gamma}) \operatorname{trace}\left(A T_{k+1} A^{\prime} P_{k \mid k}\right)+\operatorname{trace}\left(\left(D_{k+1}+(1-\bar{\gamma}) T_{k+1}\right) Q\right) \\
& +\bar{\nu} \min _{u_{k}}\left(u_{k}^{\prime}\left(U_{k}+B^{\prime}\left(S_{k+1}+(1-\bar{\nu})(1-\bar{\gamma}) T_{k+1}\right) B\right) u_{k}+\right. \\
& \left.+2 u_{k}^{\prime} B^{\prime} S_{k+1} A \hat{x}_{k \mid k}\right) .
\end{aligned}
$$

The minimizer can be easily computed, since the quantity inside the parenthesis is a convex quadratic function:

$$
\begin{aligned}
u_{k}^{*}= & -\left(U_{k}+B^{\prime}\left(S_{k+1}+(1-\bar{\nu})(1-\bar{\gamma}) T_{k+1}\right) B\right)^{-1} \\
& \cdot B^{\prime} S_{k+1} A \hat{x}_{k \mid k}=L_{k} \hat{x}_{k \mid k},
\end{aligned}
$$

which is linear function of the estimated state $\hat{x}_{k \mid k}$. Substituting back into the value function we get:

$$
\begin{aligned}
& V_{k}\left(x_{k}\right)=\mathbb{E}\left[x_{k}^{\prime}\left(W_{k}+A^{\prime} S_{k+1} A\right) x_{k} \mid \mathcal{G}_{k}\right]+ \\
& +(1-\bar{\gamma}) \operatorname{trace}\left(A T_{k+1} A^{\prime} P_{k \mid k}\right)+\operatorname{trace}\left(\left(D_{k+1}+(1-\bar{\gamma}) T_{k+1}\right) Q\right) \\
& -\bar{\nu} \hat{x}_{k \mid k} A^{\prime} S_{k+1} B L_{k} \hat{x}_{k \mid k} \\
& =\mathbb{E}\left[x_{k}^{\prime}\left(W_{k}+A^{\prime} S_{k+1} A-\bar{\nu} A^{\prime} S_{k+1} B L_{k} \mid \mathcal{G}_{k}\right]+\right. \\
& +\operatorname{trace}\left(\left((1-\bar{\gamma}) A T_{k+1} A^{\prime}+\bar{\nu} A^{\prime} S_{k+1} B L_{k} P_{k \mid k}\right)+\right. \\
& +\operatorname{trace}\left(\left(D_{k+1}+(1-\bar{\gamma}) T_{k+1}\right) Q\right)
\end{aligned}
$$

Therefore, $V_{k}\left(x_{k}\right)=\hat{x}_{k \mid k}^{\prime} S_{k} \hat{x}_{k \mid k}+\operatorname{trace}\left(T_{k} P_{k \mid k}\right)+\operatorname{trace}\left(D_{k} Q\right)$. This implies that:

$$
\begin{aligned}
& S_{k}=A^{\prime} S_{k+1} A+W_{k}-\bar{\nu} A^{\prime} S_{k+1} B . \\
& \cdot\left(U_{k}+B^{\prime}\left(S_{k+1}+(1-\bar{\nu})(1-\bar{\gamma}) T_{k+1}\right) B\right) B^{\prime} S_{k+1} A \\
& T_{k}=(1-\bar{\gamma}) A^{\prime} T_{k+1} A+A^{\prime} S_{k+1} A+W_{k}-S_{k} \\
& D_{k}=D_{k+1}+(1-\bar{\gamma}) T_{k+1}
\end{aligned}
$$

The optimal minimal cost for the finite horizon, $J_{N}^{*}=V_{0}\left(x_{0}\right)$ is then given by:

$$
J_{N}^{*}=\bar{x}_{0}^{\prime} S_{0} \bar{x}_{0}+\operatorname{trace}\left(S_{0} P_{0}\right)+(1-\bar{\gamma}) \sum_{k=1}^{N} \operatorname{trace}\left(T_{k} Q\right)(54)
$$

For the infinite horizon optimal controller, necessary and sufficient condition for the average minimal cost $J_{\infty} \triangleq \lim _{N \rightarrow+\infty} \frac{1}{N} J_{N}^{*}$ to be finite is that the coupled iterative Equations (52) and (53) should converge to a finite value $S_{\infty}$ and $T_{\infty}$ as $N \rightarrow+\infty$. In the work of Imer et al. (Imer et al., 2004) similar equations where derived for the optimal LQG control under UDP for the same framework with the additional conditions $Q=0$ and $B$ square and invertible. They find necessary and sufficient conditions for those equations to converge. Unfortunately, these conditions do not hold for the general case when $B$ in not square. This is a very frequent situation in control systems, where in general we simply have $(A, B)$ controllable. We are currently trying to derive necessary and sufficient conditions for the iterative equations to converge for the more general case when $B$ is not square and the pair $(A, B)$ is controllable.

\section{CONCLUSION AND FUTURE WORK}

(50) This paper analyzes the LQG control problem in the case where both observation and control packets may be lost when travelling through a communication channel. This is the case of many distributed systems, where sensors, controllers and actuators physically reside in different locations and they have to rely on network communication to exchange information. In this context the paper presents analysis of the LQG control problem for two types of protocols,i.e. TCP and UDP. In the first case packet acknowledgement of arrival of control packets is available to the controller, while it is not available in general in the second case. 
For TCP-like protocols a solution for a general LTI stochastic system is provided for both the finite and infinite horizon case, showing that the optimal control is still a linear function of the state. Moreover, the infinite horizon cost function $J_{\infty}$ is bounded if arrival probabilities $\bar{\gamma}, \bar{\nu}$ are higher than a specified threshold. UDP-like protocols present a much more complex scenario, as the lack of acknowledgement of the control packet at the controller makes the separation principle not valid anymore. Estimation and control are now coupled. The paper shows that in general the optimal control is non linear. The control law cannot be determined in closed form, making this solution impractical. The only case where the optimal controller is linear is the one where $C$ is invertible and there is no output noise, i.e. $R=0$. The optimal solution is presented for this case.

Future work will involve the study of convergence for this special case, where, given the particular structure of the problem, boundedness of the cost function implies convergence in the infinite horizon case. From a practical standpoint, it is useful to compute the optimal static linear control for the UDP case. Even though this constitutes a suboptimal solution for the original problem, ease of computation and implementation will make it a valuable resource for the designer.

\section{REFERENCES}

Bertsekas, D. and J. Tsitsiklis (1996). NeuroDynamic Programming. Athena Scientific.

Gupta, V., D. Spanos, B. Hassibi and R. M. Murray (2004). Optimal LQG control across a packet-dropping link. Technical report. California Institute of technology. Preprint, submitted for publication.

Hadjicostis, C. N. and R. Touri (2002). Feedback control utilizing packet dropping network links. In: Proceedings of the 41 st IEEE Conference on Decision and Control. Las Vegas, NV. Invited.

Imer, O. C., S. Yuksel and T. Basar (2004). Optimal control of dynamical systems over unreliable communication links. In: NOLCOS. Stutgart, Germany.

Kumar, P.R. and P. Varaiya (1986). Stochastic Systems: Estimation, Identification and Adaptive Control. Information and System Science Series. Prentice Hall. Englewood Cliffs, NJ 07632.

Ling, Q. and M.D. Lemmon (2003a). Optimal dropout compensation in networked control systems. In: IEEE conference on decision and control. Maui, HI.

Ling, Q. and M.D. Lemmon (2003b). Soft realtime scheduling of networked control systems with dropouts governed by a markov chain. In: American Control Conference. Denver, $\mathrm{CO}$.
Nilsson, Johan (1998). Real-Time Control Systems with Delays. PhD thesis. Department of Automatic Control, Lund Institute of Technology.

Sinopoli, B., C. Sharp, S. Schaffert, L. Schenato and S. Sastry (2003). Distributed control applications within sensor networks. IEEE Proceedings Special Issue on Distributed Sensor Networks.

Sinopoli, B., L. Schenato, M. Franceschetti, K. Poolla, M.I. Jordan and S. Sastry (2004). Kalman filtering with intermittent observations. IEEE Transactions on Automatic Control. 